\title{
AVALIAÇÃO DE FUNGICIDAS NO CONTROLE DE Asperisporium caricae NA CULTURA DO MAMOEIRO ${ }^{1}$
}

\author{
LIVIAA FELÍCIO BARRETO², PAULO ALBERTO LEGORI SAVAN 3 , \\ LONJORÉ LEOCADIO DE LIMA4, BRUNO NASCIMENTO LODO 5
}

Resumo - Dentre as doenças fúngicas do mamoeiro, a varíola (Asperisporium caricae) é umas das doenças mais importantes. Os objetivos deste trabalho foram avaliar a eficácia de fungicidas no controle da varíola em folhas e frutos do mamoeiro. O experimento foi conduzido em área comercial, no município de Taquarintiga - SP, em delineamento de blocos casualizados, com cinco tratamentos e quatro repetições. Os tratamentos foram tebuconazol + trifloxistrobina $\left(0,06 \mathrm{~L} \cdot 100 \mathrm{~L}^{-1}\right.$ de água), piraclostrobina $\left(0,04 \mathrm{~L} .100 \mathrm{~L}^{-1}\right.$ de água), difenoconazol $\left(0,03 \mathrm{~L} \cdot 100 \mathrm{~L}^{-1}\right.$ de água), azoxistrobina $\left(128 \mathrm{~g} \cdot \mathrm{ha}^{-1}\right) \mathrm{e}$ a testemunha. Foram realizadas três avaliações, nas quais foram avaliadas a severidade da varíola através de escala diagramática, nas folhas e nos frutos de três plantas previamente marcadas. Os dados registrados foram submetidos à análise de variância pelo teste F, e as médias, comparadas pelo teste de Scott-Knott $(\mathrm{p}<0,05)$. Os fungicidas tebuconazol+trifloxistrobina, piraclostrobina, difenoconazol e azoxistrobina foram eficientes no controle da varíola.

Termos para indexação: varíola; severidade; doença.

\section{EVALUATION OF FUNGICIDES TO CONTROL Asperisporium caricae IN PAPAYA CROP}

\begin{abstract}
Among the fungal diseases as smallpox (Asperisporium caricae) is the most important diseases of papaya. Objectives of this article were to evaluate the efficacy of fungicides to control smallpox papaya. The experiment was conducted in a commercial area in the municipality of Taquarintiga - SP in RBD with five treatments and four replications. The treatments were Tebuconazole + trifloxystrobin $(0.06 \mathrm{~L} / 100 \mathrm{~L})$, Pyraclostrobin (0.04 L/100L) Difenoconazol (0.03 L/100L), Azoxystrobin and a witness. Were performed 3 evaluations, which were evaluated the severity of smallpox through diagrammatic scale, on leaves and fruits of three plants previously marked and on each assessment two leaves and four fruits were chosen randomly and evaluated. The data were subjected to analysis of variance by $\mathrm{F}$ test and means compared by Scott-Knott test $(\mathrm{p}<0.05)$. The products Tebuconazol + trifloxystrobin, Pyraclostrobin, Difenoconazol and Azoxystrobin controlled smallpox, but were not effective for control of anthracnose.
\end{abstract}

Índex terms: smallpox, severity, disease.

\section{INTRODUÇÃO}

O mamoeiro (Carica papaya L.) é uma das fruteiras mais comuns em quase todos os países da américa tropical, e é considerada como uma das mais cultivadas e consumidas nas regiões tropicais e subtropicais do mundo (SERRANO; CAETANO, 2010).

O Brasil é o segundo maior produtor mundial de mamão, ficando atrás somente da Índia, com uma produção de aproximadamente dois milhões de toneladas. Bahia e Espírito Santo são os maiores estados produtores de mamão (AGRIANUAL, 2011). Nessas regiões, o mamoeiro é afetado por diferentes doenças que causam danos econômicos à cultura. Dentre as doenças fúngicas, a varíola (Asperisporium caricae) e a antracnose (Colletotrichum gloeosporioides) são as mais importantes da cultura.

A varíola é a doença mais comum do mamoeiro. Sua importância está relacionada à infecção ocorrer

\footnotetext{
${ }^{1}$ Trabalho Sinfruit 154 - Simpósio Internacional de Fruticultura - Avanços na Fruticultura (17 a 21 Outubro)

${ }^{2}$ Eng. Agr., Mestrando em Agronomia (Programa Produção Vegetal), Departamento de Produção Vegetal, UNESP/FCAV, Câmpus de Jaboticabal. Via de acesso Prof. Paulo Donato Castellane, s/n, Cep: 14884-900. E-mail: liviafbarreto@hotmail.com

${ }^{3}$ Graduando do curso de Agronomia, UNESP/FCAV, Câmpus de Jaboticabal. Via de acesso Prof. Paulo Donato Castellane, s/n, Cep: 14884-900. E-mail: Paulo.savan@hotmail.com

${ }^{4}$ Eng. Agr., Doutoranda em Agronomia (Programa Produção Vegetal), Departamento de Fitossanidade, UNESP/FCAV, Câmpus de Jaboticabal. Via de acesso Prof. Paulo Donato Castellane, s/n, Cep: 14884-900. E-mail: lonjore@hotmail.com

${ }^{5}$ Eng. Agr. MSc., (Programa Produção Vegetal), Departamento de Fitossanidade, UNESP/FCAV, Câmpus de Jaboticabal. Via de acesso Prof. Paulo Donato Castellane, s/n, Cep: 14884-900. E-mail: brunolodo@yahoo.com.br
} 
nas folhas e frutos, com consequente redução da taxa fotossintética da planta e depreciação da qualidade comercial dos frutos (REZENDE; MARTINS, 2005). De acordo com Ueno et al. 2001, apesar de ocorrer com grande frequência, é uma doença ainda pouco estudada. Santos e Barreto (2003) relataram perdas de até 30\% na comercialização do mamão no Estado de São Paulo devido a esta doença, e que, no entanto, só recentemente, se iniciaram estudos epidemiológicos sobre a doença e começaram a ser testadas estratégias de controle com diferentes fungicidas.

Os sintomas são observados tanto nas folhas como nos frutos. Na face superior das folhas, aparecem manchas normalmente circulares, pequenas, de coloração pardo-clara, circundadas por um halo amarelo, tornando-se posteriormente necróticas e brancas. Na face inferior, na região correspondente à lesão, há formação da frutificação escura do fungo. Nos frutos, os primeiros sintomas podem ser observados quando estes são pequenos e verdes, e caracterizam-se por áreas circulares encharcadas, de centro esbranquiçado, progredindo para pústulas marrons e salientes (REZENDE; MARTINS, 2005). As lesões nos frutos afetam a aparência, depreciando o valor de mercado, além de facilitar a infecção por patógenos que causam doenças de pós-colheita (VENTURA et al., 2003). Em condições favoráveis é recomendada a aplicação de fungicidas do grupo dos triazóis e das estrobilurinas (VENTURA et al., 2003). A aplicação de fungicidas ocorre de forma preventiva ou ao surgimento dos primeiro sintomas. Produtos à base de chlorotalonil + tiophanato metílico, tebuconazole, azoxystrobina e famoxadone + mancozeb demonstraram boa eficiência no controle da varíola (CELOTO et al., 2003; TATAGIBA; AGUILAR, 2005).

As medidas de controle recomendadas para a varíola são a retirada das folhas e frutos doentes do pomar, para reduzir o inóculo do fruto, aliada à aplicação de fungicidas protetores ou sistêmicos, quando há o aparecimento dos primeiros sintomas da doença (REZENDE; MARTINS, 2005). De acordo com Agrofit (2011), para o controle da varíola, estão registrados 29 produtos comerciais, com 11 princípios ativos.

Devido à importância da doença na cultura, foi objetivo deste trabalho avaliar a eficácia de fungicidas no controle da varíola do mamoeiro.

\section{MATERIAL E MÉTODOS}

O experimento foi conduzido em área comercial da cultura, plantada com a cultivar Formosa, no município de Taquarintiga - SP, georreferenciado nas coordenadas S: $21^{\circ} 24^{\prime} 24^{\prime \prime} ; \mathrm{W}: 48^{\circ} 29^{\prime} 53^{\prime \prime}$ e em uma altitude de $512 \mathrm{~m}$. Todos os tratos culturais adotados foram os recomendados para a cultura, exceto as aplicações de fungicidas.

Em todas as aplicações de fungicidas, foi acrescentado o inseticida-acaricida Clorfenapir, na dose de $30 \mathrm{~mL} .100 \mathrm{~L}-1$ de água, para o controle do ácaro-branco (nome científico) do mamoeiro.

Os tratamentos foram constituídos pelos produtos e doses relacionados na Tabela 1 .

O delineamento utilizado foi o de blocos ao acaso, com cinco tratamentos e quatro repetições. Cada parcela foi constituída de uma linha de 10 metros de comprimento, com as plantas espaçadas em 3 metros entre linhas e 2 metros entre si, totalizando $30 \mathrm{~m} 2$ por parcela e $120 \mathrm{~m} 2$ por tratamento.

As aplicações dos fungicidas foram realizadas com pulverizador montado, motorizado, com pistola e bico cônico, sendo a primeira aplicação realizada no início do aparecimento dos sintomas, e as demais, em intervalos de 14 dias, totalizando quatro aplicações, em 17 de dezembro, 05 e 19 de janeiro, e 3 de fevererio de 2011.

Foram realizadas três avaliações da severidade da varíola, em 19 de janeiro, 03 de fevereiro e 02 de março de 2011. A severidade, tanto nas folhas como nos frutos, foi avaliada a partir do início dos primeiros sintomas, em três plantas previamente marcadas. Nessas plantas, marcou-se a primeira folha sem sintomas, e foram avaliadas duas folhas acima desta, totalizando seis folhas por repetição.

As avaliações da severidade da varíola nas folhas foram realizadas com auxílio de escala diagramática (Figura 1), expressas em porcentagens de área foliar lesionada, em que: $0=$ folha sem lesões; $1=$ até $0,5 \% ; 2=0,6$ a $3 \% ; 3=3,1 \%$ a $12,5 \%$; $4=12,6 \%$ a $25 \% ; 5=25,1 \%$ a $35 \% ; 6=\mathrm{com}$ mais de $35,1 \%$.

Os frutos também foram avaliados, utilizando escala diagramática de severidade da doença (Figura 2), de acordo com Vivas et al. 2010, em que: os níveis de 0,$1 ; 0,3 ; 0,6 ; 1,2 ; 2,5 ; 5,0 ; 10$ e $20 \%$ correspondem à área superficial lesionada.As notas atribuídas às folhas e frutos foram convertidas em porcentagens de área afetada, substituindo-se a nota pelo valor médio do intervalo considerado em cada nota. Os dados obtidos foram submetidos à análise de variância, pelo teste $\mathrm{F}$, e as médias, comparadas pelo teste de Scott-Knott, ao nível de 5\% de probabilidade.

\section{RESULTADOS E DISCUSSÃO}

Os valores da comparação das médias de severidade da varíola nas folhas e nos frutos do mamoeiro 
estão apresentados na Tabela 2. Com base nestes dados, observou-se que, na primeira avaliação da severidade da varíola nos frutos, não houve diferença significativa entre os tratamentos.

$\mathrm{Na}$ segunda avaliação da severidade da varíola nos frutos, houve diferença significativa entre os tratamentos. A utilização de azoxistrobina não apresentou diferença significativa da testemunha sem aplicação, correspondendo a, respectivamente, $0,90 \%$ e $1,03 \%$ de áreas superficiais do fruto afetadas pela doença. A aplicação de tebuconazol + trifloxistrobina, piraclostrobina e difenoconazol não apresentaram diferença significativa entre si, porém diferiram das demais. No entanto, na terceira avaliação, todos os tratamentos controlaram bem a varíola nos frutos diferindo-se apenas da testemunha. Nas folhas, os tratamentos que mostraram melhor controle em praticamente todas as avaliações foram o tebuconazol+trifloxistrobina, piraclostrobina e difenoconazol (Tabela 2). Vawdrey et al. (2008), avaliando o efeito de fungicidas para o controle da varíola, constataram que piraclostrobina e o difenoconazol, isolados ou em programas de pulverização, foram eficientes no controle da doença corroborando os dados deste trabalho.

Dianese et al. (2008), avaliando o efeito de fosfitos na redução da varíola, em campo e telado, concluíram que embora todos os produtos testados tenham reduzido a severidade da varíola significativamente, quando comparados ao controle, tanto as folhas quanto os frutos apresentaram sintomas típicos da doença, assim como os produtos testados neste trabalho, porém a tolerância ao dano causado por doenças em frutos destinados ao mercado internacional é extremamente baixa, e, às vezes, uma única lesão é o bastante para rejeitar um lote.

Portanto, todos os tratamentos controlaram a varíola tanto nas folhas como nos frutos, porém os tratamentos mais eficientes em todas as avaliações foram o tebuconazol+trifloxistrobina, piraclostrobina e difenoconazol.

TABELA 1 - Fungicidas utilizados para avaliar a eficiência no controle da varíola do mamoeiro em Taquaritinga-SP.

\begin{tabular}{|c|c|c|c|c|}
\hline Princípio Ativo & & Modo de ação & Formulação & $\begin{array}{c}\text { Dose (L.100L } \\
\text { de água) }\end{array}$ \\
\hline Tebuconazol+Trifloxistrobina & & Mesostêmico Sistêmico & $\begin{array}{c}\text { Suspensão Con- } \\
\text { centrada }\end{array}$ & 0,06 \\
\hline Piraclostrobina & Sistêmico & $\begin{array}{c}\text { Concentrado } \\
\text { Emulsionável }\end{array}$ & 0,04 \\
\hline Difenoconazol & & Sistêmico & $\begin{array}{c}\text { Concentrado } \\
\text { Emulsionável }\end{array}$ & 0,03 \\
\hline Azoxistrobina & & Sistêmico & $\begin{array}{c}\text { Granulado Disper- } \\
\text { sível }\end{array}$ & 128 g.ha $^{-1}$ \\
\hline Testemunha & - & - & - & - \\
\hline
\end{tabular}

TABELA 2 - Efeito de fungicidas aplicados em mamoeiros. Taquaritinga-SP, 2011.

\begin{tabular}{lrrrrrr}
\hline \multirow{2}{*}{ Tratamentos } & \multicolumn{3}{c}{ Frutos $^{* *}$} \\
\cline { 2 - 7 } & $\mathbf{1 9 / 0 1}$ & $\mathbf{0 3 / 0 2}$ & $\mathbf{0 2 / 0 3}$ & $\mathbf{1 9 / 0 1}$ & $\mathbf{0 3 / 0 2}$ & $\mathbf{0 2} / \mathbf{0 3}$ \\
\hline Tebuconazol + Trifloxistrobina & $0,008 \mathrm{a}$ * & $0,13 \mathrm{~b}$ & $0,20 \mathrm{~b}$ & $0,23 \mathrm{c}$ & $0,33 \mathrm{~b}$ & $0,70 \mathrm{c}$ \\
Piraclostrobina & $0,025 \mathrm{a}$ & $0,33 \mathrm{~b}$ & $0,16 \mathrm{~b}$ & $0,31 \mathrm{c}$ & $0,22 \mathrm{~b}$ & $2,72 \mathrm{c}$ \\
Difenoconazol & $0,014 \mathrm{a}$ & $0,10 \mathrm{~b}$ & $0,29 \mathrm{~b}$ & $0,44 \mathrm{c}$ & $0,52 \mathrm{~b}$ & $3,35 \mathrm{c}$ \\
Azoxistrobina & $0,012 \mathrm{a}$ & $0,90 \mathrm{a}$ & $0,46 \mathrm{~b}$ & $1,02 \mathrm{~b}$ & $0,91 \mathrm{~b}$ & $6,85 \mathrm{~b}$ \\
Testemunha & $0 \mathrm{a}$ & $1,03 \mathrm{a}$ & $0,84 \mathrm{a}$ & $1,75 \mathrm{a}$ & $6,95 \mathrm{a}$ & $19,68 \mathrm{a}$ \\
\hline Coeficiente de Variação (\%) & 1,95 & 17,06 & 8,80 & 14,87 & 17,47 & 19,36 \\
\hline
\end{tabular}

*Médias seguidas de mesma letra na coluna não diferem estatisticamente entre si, pelo teste de Scott Knott, a 5 \% de probabilidade

$* *$ Previamente às análises, os dados de severidade foram transformados para $\sqrt{x}+0,5$. 


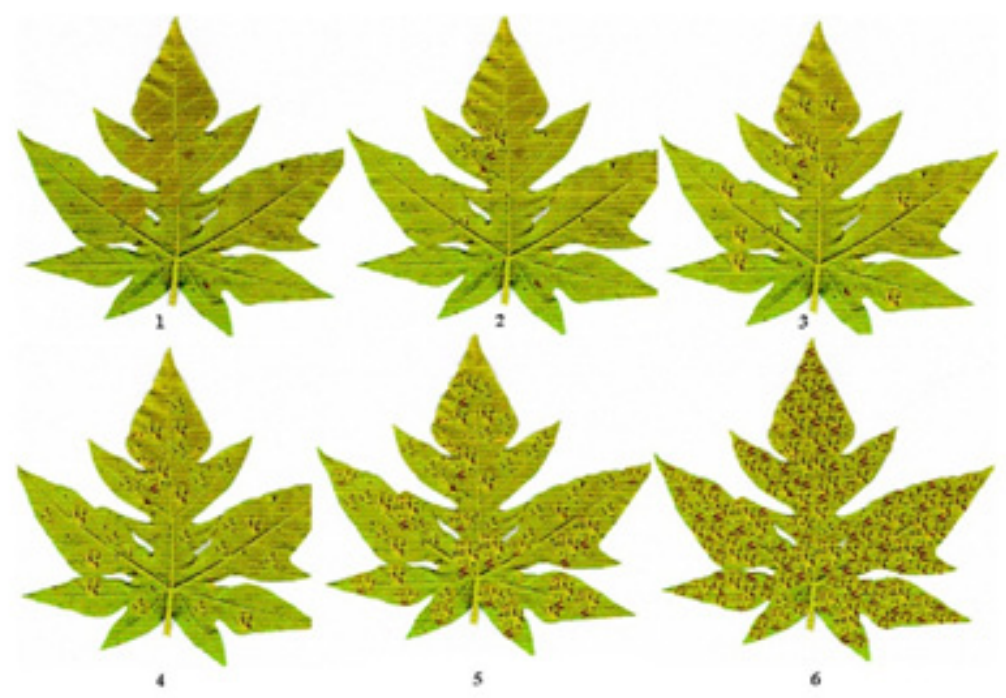

FIGURA 1: Escala de notas para avaliação da severidade da variola em folhas de mamoeiro.
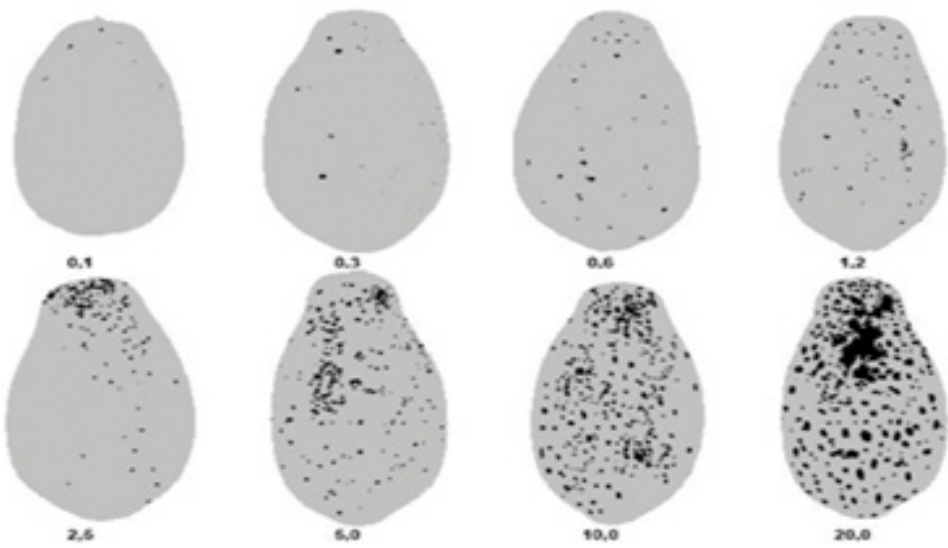

FIGURA 2: Escala de notas para avaliação da severidade da variola em frutos de mamoeiro.

\section{CONCLUSÃO}

Os fungicidas tebuconazol+trifloxistrobina, piraclostrobina, difenoconazol e azoxistrobina controlam a varíola do mamoeiro.

\section{REFERÊNCIAS}

AGRIANUAL 2011: anuário da agricultura brasileira. São Paulo: AgraFNP, 2011. p. 325 - 332.

AGROFIT 2011. Disponível em: <http:/extranet. agricultura.gov.br/agrofit_cons/principal_agrofit_cons. Acesso em: 24 maio 2011.
CELOTO, M. I. B.; PAPA, M. F. S.; CORREA, L. S. Efeito de fungicidas no controle da varíola (Asperisporium caricae) do mamoeiro. Summa Phytopathologica, Botucatu, v. 29, p. 87, 2003.

DIANESE, A. C.; BLUM, L. E.B.; DUTRA, J,J.; LOPES, L. F.; SENA, M. C.; FREITAS, L. F. Avaliação do efeito de fosfitos na redução da varíola (Asperisporium caricae) do mamoeiro (Carica papaya). Revista Brasileira de Fruticultura, Jaboticabal, v. 30, n. 3. 2008 . 
REZENDE, J.A.M.; MARTIN, M.C. Doenças do mamoeiro. In: KIMATI, H.; AMORIM, L.; REZENDE, J.A.M.; BERGAMIN FILHO, A.; CAMARGO, L.E.A. Manual de fitopatologia: doenças de plantas cultivadas. São Paulo: Agronômica Ceres, 2005. v.2, cap. 49, p. $436-443$.

SANTOS, M.C.; BARRETO, M. Estudo epidemiológico da varíola do mamoeiro em cultivares submetidas a tratamentos com fungicidas. Summa Phytopathologica, Botucatu, v. 29, p. 141-146. 2003.

SERRANO, L. A. L.; CATTANEO, L. F. O cultivo do mamoeiro no Brasil. Revista Brasileira de Fruticultura, Jaboticabal, v. 32, n. 3. 2010.

TATAGIBA, J. S.; AGUILAR, M.A.G. Eficiência do Midas BR no controle da pinta-preta (Asperisporium caricae) do mamoeiro (Carica papaya L.). In: MARTINS, D. S. (Ed.). Papaya Brasil: mercado e inovações tecnológicas para o mamão. Vitória: Incaper, 2005. p. 405-408.
UENO, B.; FERREIRA, M.A.S.V.; UESUGI, C.H.L. Levantamento das principais doenças de mamoeiro (Carica papaya L.) na região de Barreiras-BA. Fitopatologia Brasileira, Brasília, v. 26, p. 386, 2001.

VAWDREY, L.L.; GRICE, K.R.E.; WESTERHUIS, D. Field and laboratory evaluations of fungicides for the control of brown spot (Corynespora cassiicola) and black spot (Asperisporium caricae) of papaya in far north Queensland, Australia. Australasian Plant Pathology, Auckland, v. 37, n. 6, p. 552-558, 2008.

VENTURA, J.A.; COSTA, H.; TATAGIBA,J.S. Manejo das doenças do mamoeiro. In: MARTINS, D.S.; COSTA, A. F. S. (Ed.). A cultura do mamoeiro: tecnologias de produção. Vitória, Incaper, 2003. p. 231-310.

VIVAS, M.; TERRA, C.E.P.S.; SILVEIRA, S.F.; FONTES, R.V.; PEREIRA, M.G. Escala diagramática para avaliação da severidade de pinta-preta em frutos de mamoeiro. Summa Phytopathologica, Botucatu, v.36, n.2, p.161-163. 2010. 